\title{
Three-dimensional crystal structure of the A-tract DNA dodecamer d(CGCAAATTTGCG) complexed with the minor-groove-binding drug Hoechst 33258
}

\author{
M. Cristina VEGA ${ }^{1,2}$, Isabel GARCÍA SÁEZ ${ }^{1,2}$, Joan AYMAMÍ ${ }^{2}$, Ramón ERITJA ${ }^{1}$, Gijs A. VAN DER MAREL ${ }^{3}$, \\ Jaques H. VAN BOOM ${ }^{3}$, Alexander RICH ${ }^{4}$ and Miquel COLL ${ }^{1.2}$ \\ ${ }^{1}$ Departament de Biologia Molecular i Cel-lular, Centre d'Investigació i Desenvolupament-C. S. I. C., Barcelona, Spain \\ 2 Departament d'Enginyeria Química, Universitat Politècnica de Catalunya, Barcelona, Spain \\ ${ }^{3}$ Gorlaeus Laboratory, Leiden State University, The Netherlands \\ ${ }^{4}$ Department of Biology, Massachusetts Institute of Technology, Cambridge MA, USA
}

(Received February 15/March 24, 1994) - EJB 94 0220/2

The molecular structure of the DNA A-tract dodecamer d(CGCAAATTTGCG) complexed with the drug Hoechst 33258 has been determined by X-ray diffraction analysis. The Hoechst molecule binds in the DNA minor groove covering the sequence AATTT of the central A-tract, with the piperazine group close to one of the GC regions. The drug molecule makes two three-centered hydrogen bonds from the nitrogen atoms of the benzimidazole rings to the $\mathrm{N} 3$ and $\mathrm{O} 2$ atoms of the DNA bases. Although a high propeller twist is observed in the A-tract, only one unsymmetrical three-centered hydrogen bond is present in the DNA major groove. The structure is compared with other minor-groove-binding drug complexes and the influence of these drugs on DNA A-tracts is discussed.

Hoechst 33258 [chemical name: 2'-(4-hydroxyphenyl)5-(4-methyl-1-piperazinyl)-2,5'-bi-benzimidazole], netropsin and distamycin are DNA minor-groove-binding drugs with common structural features: (a) they are molecules containing planar segments due to the presence of aromatics groups; (b) they are non-symmetrical molecules; (c) they are positively charged and (d) they have an arc-like shape which allows them to follow the minor groove when interacting with the DNA double helix. Furthermore, these molecules have affinity for $A+T$-rich sequences over $\mathrm{G}+\mathrm{C}$-containing sequences mostly due to steric factors (Burckhardt et al., 1985). The presence of the N2 amino group in guanine prevents the binding of the drug in the DNA minor groove (Kopka et al., 1985), although Hoechst 33258 tolerates G-C pairs at the edge of the binding site (Harshman and Dervan, 1985; Portugal and Waring, 1988).

Hoechst 33258 is a synthetic $N$-methyl piperazine derivative with two benzimidazole groups and one phenyl group (Fig. 1). It has antihelminthic activity (Lammler et al., 1971) and is commonly used as a fluorescent cytological stain for DNA (Hilwig and Gropp, 1972; Holmquist, 1975). Hoechst 33258 binds preferentially to $\mathrm{A}+\mathrm{T}$-rich regions as determined by fluorescence (Latt and Wohlleb, 1975; Holmquist, 1975), footprinting (Harshman and Dervan, 1985) and ${ }^{125}{ }^{-}$DNA cleavage (Martin and Holmes, 1983; Murray and Martin, 1988).

There are several X-ray diffraction studies of this drug complexed with two different dodecamers. The complex Hoechst 33258-d(CGCGAATTCGCG) has been reported by

Correspondence to M. Coll, Departament de Biologia Molecular i Cel-lular, Centre d'Investigació i Desenvolupament-C. S. I. C., Jordi Girona 18, E-08034 Barcelona, Spain

Abbreviation. Hoechst 33258, 2'-(4-hydroxyphenyl)-5-(4-methyl-1-piperazinyl)-2,5'-bi-benzimidazole.

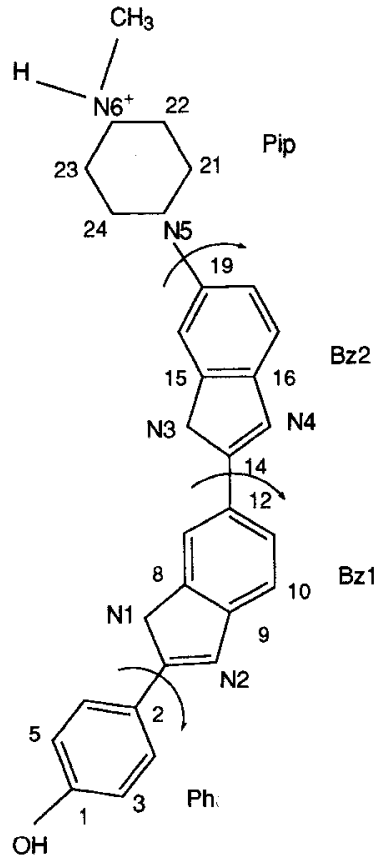

Fig. 1. Atomic numbering scheme of the Hoechst 33258 molecule. The drug has four structural groups: a phenyl ring $(\mathrm{Ph})$, two benzimidazole rings ( $\mathrm{Bz} 1$ and $\mathrm{Bz} 2$ ) and a piperazine ring (Pip). The torsion angles between the four structural groups are indicated with arrows. The N1 and N3 atoms interact with the bases at the floor of the DNA minor groove.

Pjura et al. (1987), Teng et al. (1988) and Quintana et al. (1991). The different analyses show some discrepancies in the positioning of the drug along the minor groove which might be due to different crystallization conditions or dif- 
ferent interpretations of the electron density maps. The complex of Hoechst 33258 with the alternating sequence d(CGCGATATCGCG) has been studied by Carrondo et al. (1989). Recently Sriram et al. (1992) have reported the complex with the dodecamer d(CGC $\left[e^{6} \mathrm{G}\right]$ AATTCGCG), where $\mathrm{e}^{6} \mathrm{G}$ stands for $O^{6}$-ethylguanine. In all these complexes the DNA sequence has four $\mathrm{A} \cdot \mathrm{T}$ base pairs which is approximately the length of the Hoechst dye. However the drug molecule does not bind in the same position when the central sequence is AATT or ATAT. A sequence-dependent specificity seems to drive the drug binding.

In this paper we report the structural analysis of the complex of Hoechst 33258 with the DNA dodecamer d(CGCAAATTTGCG). In this dodecamer the central $A \cdot T$ region is longer than the length of the drug and therefore several binding areas are possible along the A-tract. In the complex structure of the same dodecamer with the drugs distamycin and netropsin we have observed a single binding site (or at least a major site) rather than a disorder between several positions along the groove (Coll et al., 1987; Tabernero et al., 1993).

We wanted to test, with a different drug, if this is a general characteristic of minor-groove-binders. In addition, the complex reported here is compared with other Hoechst 33258-DNA complexes and the influence of drug binding on the central A-tract is analyzed.

\section{EXPERIMENTAL PROCEDURES}

The DNA dodecamer d(CGCAAATTTGCG) was synthesized and purified as described (van der Marel et al., 1981). Suitable crystals of the complex were grown by vapour diffusion at room temperature from sittings drops containing $1 \mathrm{mM}$ DNA dodecamer, re-purified from previous crystallization drops by HPLC, $1 \mathrm{mM}$ Hoechst-33258, $19.5 \% 2$ methyl-2,4-pentanediol, $1 \mathrm{mM}$ sodium cacodylate $\mathrm{pH}=7$ and $0.7 \mathrm{mM}$ spermine, equilibrating against a reservoir of 50\% 2-methyl-2,4-pentanediol.

A crystal of approximate dimensions $0.2 \times 0.35 \times 0.5 \mathrm{~mm}$ was mounted in a glass capillary with a droplet of mother liquor. The crystal was orthorhombic, space group $\mathbf{P} 22_{1} 2_{1} 2_{1}$, with unit cell dimensions $a=2.55 \mathrm{~nm}, b=4.15 \mathrm{~nm}$ and $c=6.53 \mathrm{~nm}$, thus being isomorphous with the original d(CGCGAATTCGCG) dodecamer (Wing et al., 1980). Three-dimensional diffraction data were collected up to 0.24-nm resolution, at room temperature, using a Mar Research imaging plate area detector installed on an Elliot GX21 rotating anode $\mathrm{X}$-ray generator.

Images were taken in $5^{\circ}$ rotation steps with the detector set at $110 \mathrm{~mm}$ distance. The data were processed with the MOSFLM package (Leslie, 1991). A total of 9285 reflections were measured of which 2291 were considered unique above the $2(F)$ level and the $R$.
Table 1. Completeness of data for the Hoechst 33258-d(CGCAAATTTGCG) complex $\left[F_{0}>2 \sigma\left(F_{0}\right)\right]$.

\begin{tabular}{ll}
\hline Resolution range & Comp \\
\hline $\mathrm{nm}$ & \\
$5.00-0.40$ & 0.95 \\
$0.40-0.35$ & 0.94 \\
$0.35-0.30$ & 0.89 \\
$0.30-0.27$ & 0.83 \\
$0.27-0.26$ & 0.76 \\
$0.26-0.25$ & 0.69 \\
$0.25-0.24$ & 0.52 \\
\hline
\end{tabular}

corresponding to the drug molecule. The Hoechst molecule was fitted into the electron density using the computer graphics program TOM-FRODO (Cambillau and Horjales, 1987; Jones, 1978). Hoechst 33258 is a non-symmetrical molecule and consequently the existence of two possible orientations in the crystal was considered. Only in one orientation could the drug be fitted completely in the electron density with the bulkier piperazine ring positioned at one end of the A-tract.

The structure was further refined and solvent molecules were added. The location of the drug molecule was confirmed by calculating an omit map after a refinement run excluding the drug atoms (Fig. 2). The final $R$-factor is $20 \%$ for 2253 reflections between $1.0-0.24 \mathrm{~nm}$. In the final structure the root-mean-square deviation from target values is $0.0019 \mathrm{~nm}$ for bond lengths and $3.71^{\circ}$ for bond angles. The atomic coordinates have been deposited with the Brookhaven Protein Data Bank.

Empirical force-field calculations were performed for determining $\mathrm{H}$-bond interactions using a dielectric constant $\varepsilon=$ 1r. All parameters were taken from the Amber data base (Weiner et al., 1986). Angles between mean planes of the drug were calculated with the program QUEST written by G. Quigley. DNA parameters were calculated with the program NEWHEL92 written by R. Dickerson.

\section{RESULTS AND DISCUSSION}

\section{Drug-DNA interactions}

Figs 2 and 3 show the final structure of the complex with the drug molecule inserted into the minor groove of the DNA double helix. The drug molecule covers approximately five of the six central A - T base pairs from A5 - T20 to T9 A16. It is therefore displaced to one end of the A-tract with the piperazine ring positioned between the T9 . A16 base pair and $\mathrm{G} 10$. C15 base pair. In this area, the minor groove 
Table 2. Drug-DNA hydrogen bond distance in the Hoechst 33258-d(CGCAAATTTGCG) complex.

Lnerhst 22058-atop

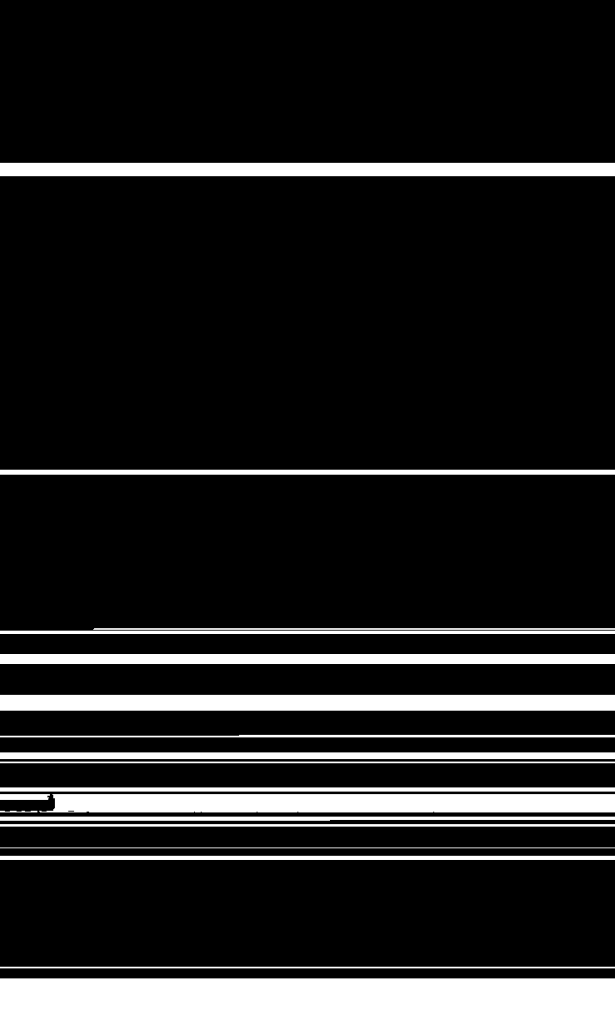


A
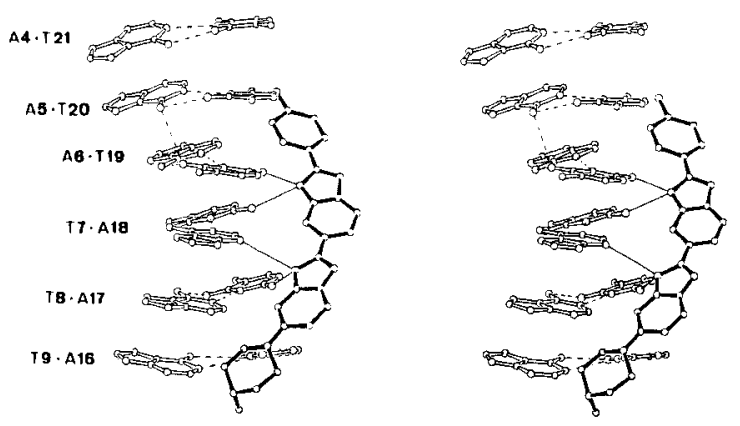

D

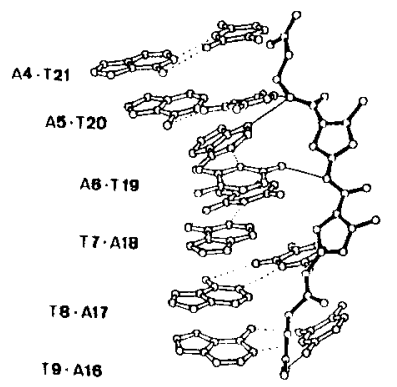

$$
\text { (1) }
$$

E

B

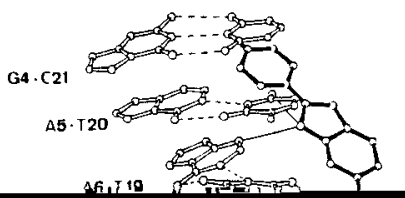

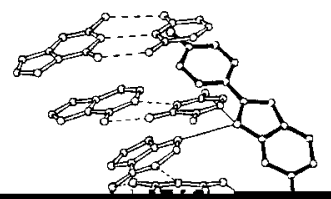

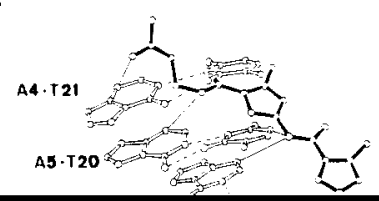

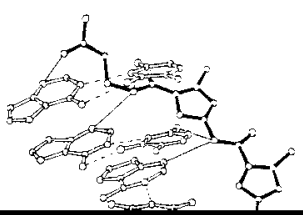




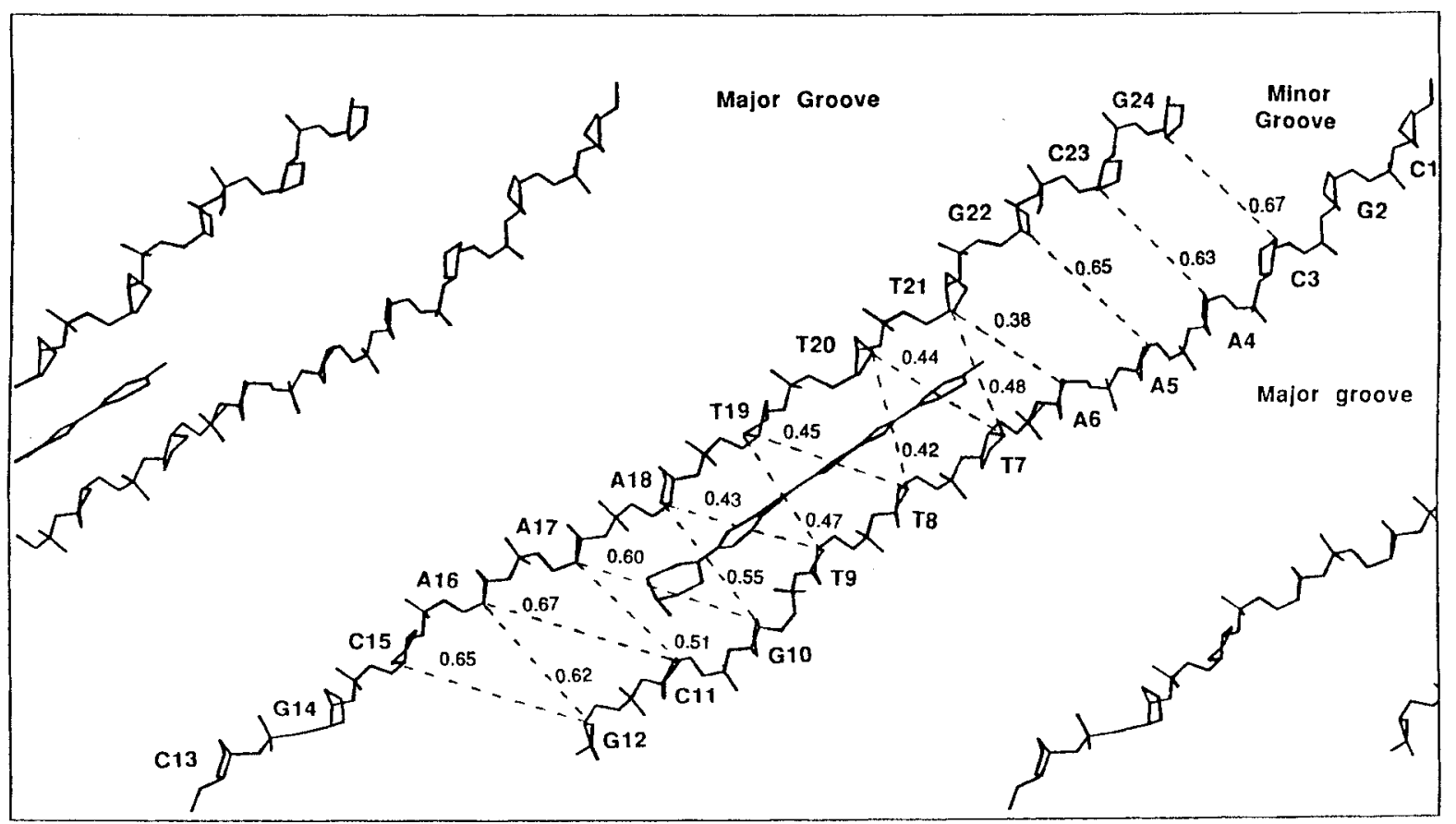

Fig. 5. Cylindrical projection of the Hoechst-d(CGCAAATTTGCG) complex. DNA bases are not plotted for clarity. The shortest O4'$\mathrm{O} 4^{\prime}$ distances across the minor groove (less $0.28 \mathrm{~nm}$ to account for the van der Waals radii of the oxygen atoms) are indicated by dashed lines and marked in $\mathrm{nm}$. The puckered ring of the piperazine moiety lies at the end of the A-tract where the minor groove opens up between A18/G10.

\section{DNA conformation}

A-tract sequences have been found to have a high propel- the native dodecamer. As the asymmetry of the bifurcated bond increases (distamycin $<$ netropsin $<$ hoechst $<$ native) the interaction becomes weaker. 
are basically the orientation of the planes of the bases in the DNA (propeller and buckle), and the torsion angles between the planar groups in the drug.

This work was supported by grants from the Comision Interministerial de Ciencia y Tecnología (SAL90-0824), the Dirección General de Investigación Científica y Técnica (PB92-0117) and the Centre de Supercomputació de Catalunya to M. Coll. M. C. V. acknowledges a fellowship from the Generalitat de Catalunya. I. G.S. acknowledges a Ministerio de Educación y Ciencia fellowship. We thank C. Aleman for helping with the H-bond energy calculations.

\section{REFERENCES}

Brown, D. G., Sanderson, M. S., Garman, E. \& Neidle S. (1992) Crystal structure of a berenyl-d(CGCAAATTTGCG) complex, J. Mol. Biol. 226, 481-490.

Brünger, A. T. (1992) XPLOR, Version 3.1, a system for X-ray crystallography and NMR, Yale University Press, New Haven and London.

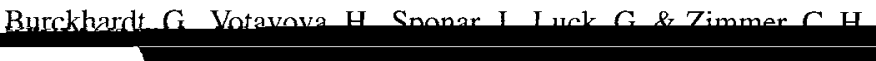

Latt, S. A. \& Wohlleb, J. C. (1975) Optical studies of interaction Hoechst 33258 with DNA, chromatin, and metaphase chromosomes, Chromosoma 52, 297-316.

Leslie, A. G. W. (1991) Recent changes to the MOSFLM package for processing film and image plate data, Daresbury Lab. Inf. Quart. Prot. Crystallogr. 26 (available from the Librarian, SERC Laboratory, Daresbury, Warrington W A4 AD, England).

Martin, R. F. \& Holmes, N. (1983) Use of an ${ }^{125}$ I-labelled DNA ligand to probe DNA structure, Nature $302,452-454$.

Murray, V. \& Martin, R. F. (1988) Sequence specificity of ${ }^{125}$ I-labelled Hoechst 33258 damage of six closely related DNA sequences, J. Mol. Biol. 203, 63-73.

Nelson, H. C. M., Finch, J. T., Luisi, F. B. \& Klug, A. (1987) The structure of an oligo(dA) - oligo(dT) tract and its biological implications, Nature 330, 221-226.

Pjura, P. E., Grzeskoviak, K. \& Dickerson, R. E. (1987) Binding of Hoechst 33258 to the minor groove of B-DNA, J. Mol. Biol. $197,257-271$.

Portugal, J. \& Waring, M. J. (1988) Assignment of DNA-binding sites for 4',6'-diamidine-2-phenylimidazole and bisbenzamidine (Hoechst 33258), a comparative footprinting study, Biochim. Biophys. Acta 949, 158-168.

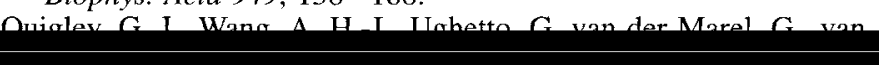

